\title{
A large and persistent outbreak of typhoid fever caused by consuming contaminated water and street-vended beverages: Kampala, Uganda, January - June 2015
}

Steven Ndugwa Kabwama ${ }^{1 *}$ (D, Lilian Bulage ${ }^{1}$, Fred Nsubuga', Gerald Pande ${ }^{1}$, David Were Oguttu', Richardson Mafigiri ${ }^{1}$, Christine Kihembo ${ }^{1}$, Benon Kwesiga ${ }^{1}$, Ben Masiira ${ }^{1}$, Allen Eva Okullo ${ }^{1}$, Henry Kajumbula ${ }^{2}$, Joseph Matovu ${ }^{3}$, Issa Makumbi ${ }^{4}$, Milton Wetaka ${ }^{4}$, Sam Kasozi ${ }^{4}$, Simon Kyazze ${ }^{4}$, Melissa Dahlke ${ }^{4}$, Peter Hughes ${ }^{5}$, Juliet Nsimire Sendagala ${ }^{5}$, Monica Musenero ${ }^{6}$, Immaculate Nabukenya $^{6}$, Vincent R. Hill', Eric Mintz', Janell Routh ${ }^{7}$, Gerardo Gómez ${ }^{7}$, Amelia Bicknese ${ }^{7}$ and Bao-Ping Zhu ${ }^{7,8}$

\begin{abstract}
Background: On 6 February 2015, Kampala city authorities alerted the Ugandan Ministry of Health of a "strange disease" that killed one person and sickened dozens. We conducted an epidemiologic investigation to identify the nature of the disease, mode of transmission, and risk factors to inform timely and effective control measures.

Methods: We defined a suspected case as onset of fever $\left(\geq 37.5^{\circ} \mathrm{C}\right)$ for more than 3 days with abdominal pain, headache, negative malaria test or failed anti-malaria treatment, and at least 2 of the following: diarrhea, nausea or vomiting, constipation, fatigue. A probable case was defined as a suspected case with a positive TUBEX ${ }^{\circledR}$ TF test. A confirmed case had blood culture yielding Salmonella Typhi. We conducted a case-control study to compare exposures of 33 suspected case-patients and 78 controls, and tested water and juice samples.

Results: From 17 February-12 June, we identified 10,230 suspected, 1038 probable, and 51 confirmed cases. Approximately $22.58 \%$ (7/31) of case-patients and 2.56\% (2/78) of controls drank water sold in small plastic bags $\left(\mathrm{OR}_{\mathrm{M}-\mathrm{H}}=8.90 ; 95 \% \mathrm{Cl}=1.60-49.00\right) ; 54.54 \%(18 / 33)$ of case-patients and $19.23 \%(15 / 78)$ of controls consumed locallymade drinks $\left(\mathrm{OR}_{\mathrm{M}-\mathrm{H}}=4.60 ; 95 \% \mathrm{Cl}: 1.90-11.00\right)$. All isolates were susceptible to ciprofloxacin and ceftriaxone. Water and juice samples exhibited evidence of fecal contamination.
\end{abstract}

Conclusion: Contaminated water and street-vended beverages were likely vehicles of this outbreak. At our recommendation authorities closed unsafe water sources and supplied safe water to affected areas.

Keywords: Typhoid fever, Outbreak, Case-control, Uganda

\section{Background}

Typhoid fever is a systemic disease caused by Salmonella enterica serovar Typhi, a Gram-negative bacterium. Humans are the only host, and transmission most commonly occurs through ingestion of water or food contaminated by feces from an acutely ill or convalescent patient or an asymptomatic carrier. The incubation

\footnotetext{
* Correspondence: skabwama@musph.ac.ug

'Uganda Public Health Fellowship Program, Field Epidemiology Track,

Ministry of Health, Kampala, Uganda

Full list of author information is available at the end of the article
}

period is usually 1 to 2 weeks but can range from 3 to 60 days [1]. The illness presents with sustained fever and a constellation of other symptoms including dry cough, fatigue, abdominal pain, diarrhea, and constipation [2]. Case fatality ratios range between 10 and $30 \%$ if untreated, but fall to $1-4 \%$ with appropriate and timely antimicrobial treatment [3]. The gold standard laboratory diagnosis of typhoid fever requires isolation of $S$. Typhi from blood, stool, bone marrow, or other tissue or bodily fluid by bacterial culture [2]. Other tests with 
moderate sensitivity and specificity include the Widal test and TUBEX ${ }^{\circ}$ TF test which involve detection of antibodies against $S$. Typhi antigens [2]. Typhoid fever is preventable through public health interventions such as provision of safe water, ensuring proper sanitation and waste disposal systems, and excluding disease carriers from handling food [4].

Typhoid fever is a major cause of mortality and morbidity worldwide. In endemic areas, the disease is most commonly found in children 5-19 years of age. International visitors from non-endemic areas are also at risk if unvaccinated [1]. The global burden of the disease in low- and middle-income countries in 2010 was estimated to be 11.9 million cases, including 129,000 fatalities, after adjusting for water-related risk factors [5]. In Uganda, an outbreak of typhoid fever in Kasese District sickened 8092 persons from 27 December 2007 to 30 July 2009, resulting in at least 249 intestinal perforations and 47 deaths [6]. In 2011, numerous typhoid cases were again reported in Kasese and neighboring Bundibugyo District with many more intestinal perforations and emergence of multidrug resistant strains [7].

On 6 February, 2015, the Ugandan Ministry of Health $(\mathrm{MoH})$ received a report from the Kampala Capital City Authority that a 42-year-old man had died a day earlier of a "strange illness." The patient was admitted to the hospital on 2 February 2015 with symptoms of abdominal pain, high fever, and severe jaundice. Initial testing involved use of the Widal test which was positive. Approximately 30 other people who worked in the same area as the deceased reportedly had similar symptoms. We conducted an epidemiologic investigation to identify the nature of the disease, mode of transmission, and risk factors to inform timely and effective control measures.

\section{Methods}

\section{Study sites}

The outbreak occurred in Kampala (estimated population: 1.4 million), the capital of Uganda [8]. Kampala has five divisions: Kampala Central, Kawempe, Makindye, Rubaga, and Nakawa. We focused our epidemiologic investigation on two markets and a commuter taxi park in Kampala Central Division where the initial cases were concentrated.

\section{Surveillance}

To characterize and control the epidemic, $\mathrm{MoH}$ conducted surveillance at six treatment centers established in affected areas of the city to provide diagnostic testing and typhoid fever treatment free of charge. These treatment centers were existing health centers in which routine disease surveillance and treatment activities are conducted. Through the media, local leaders encouraged the people with symptoms of typhoid fever to seek medical care at these treatment centers.
We defined a suspected case as onset of fever $\left(\geq 37.5^{\circ} \mathrm{C}\right)$ for $\geq 3$ days from 1 January 2015 onwards, with headache, abdominal pain, a negative test for malaria or failure to respond to anti-malaria treatment, and $\geq 2$ of the following symptoms: diarrhea, nausea or vomiting, constipation, or fatigue. A probable case was a suspected case whose serum sample yielded a positive TUBEX $^{\circ}$ TF test [9]. Blood samples were collected from the first 5 suspected cases every day from each treatment center and referred to the microbiology laboratory at the Medical Research Council for blood culture. A confirmed case was a suspected case whose blood culture yielded $S$. Typhi.

\section{Case-control study}

We conducted open-ended hypothesis-generating interviews of case-patients found in the areas where the initial cases were identified, focusing on their usual sources of water and food. To test the hypotheses generated from the interviews, we conducted a case-control study from 10 to 20 February 2015. To rapidly identify the mode of transmission so as to inform prompt prevention and control measures, we used the initial 33 suspected case-patients identified in the earliest-affected communities for the case-control study. The earliest cases were persons working in two markets or in the central terminal station for Kampala's shared taxis, all of which were located in central Kampala. Therefore we recruited both the cases and the controls from those places. The markets are open spaces where people set up their stalls to sell assorted merchandise, whereas the central terminal station for the shared taxis is an area where the shared taxis (mini-vans) pick up and drop off passengers. In the markets, after identifying and interviewing a case, the interviewer then walked around the stall to identify several persons of the same gender and similar age as the case from the surrounding stalls who never had a febrile disease since January 1, 2015, and recruited those persons as controls. Similarly, in the central terminal station for the shared taxis, after identifying and interviewing a case who was working inside a shared taxi (e.g., a driver or conductor), the interviewer then walked around the shared taxi to recruit asymptomatic workers of the same gender and similar age from the surrounding shared taxis as controls. The interviewers used a structured questionnaire to collect information on the usual water and food exposures from the case- and control-persons. A link to the questionnaire that was used has been provided in the Additional file 1 of the manuscript.

\section{Clinical laboratory investigation}

The TUBEX ${ }^{\circ}$ TF test was performed at the treatment centers by trained clinical and laboratory staff as per the manufacturer's instructions. Blood culture was 
performed on the first five patients presenting each day at the 6 treatment centers. From each adult patient, $5-10 \mathrm{~mL}$ of blood was collected and inoculated in BD Bactec $^{\mathrm{Tm}}$ Aerobic/F blood culture bottles and incubated in a BD Bactec 9000 series $^{\mathrm{rm}}$. Presumptive positive bottles, as signaled by the system, were subcultured on MacConkey, chocolate, and blood agar plates and incubated aerobically at $37^{\circ} \mathrm{C}$ for $24 \mathrm{~h}$. A Gram stain was also performed. Negative vials were incubated for up to 7 days and if the system still indicated negative, a Gram stain was performed and a final subculture was done before reporting the specimen as negative. Oxidase-negative, lactose non-fermenting colonies, were screened using API $10 \mathrm{~S}$ at the start of the outbreak. Later an abbreviated panel of biochemical tests [10] was used. Isolates biochemically typical of $S$. Typhi were serotyped using slide agglutination with $S$. polyvalent $\mathrm{O}, S$. polyvalent $\mathrm{H}, S$. O factor 9 (group D), $S$. H factor d and $S$. $\mathrm{Vi}$ antisera.

A set of $30 \mathrm{~S}$. Typhi isolates were sent to the U.S. Centers for Disease Control and Prevention (CDC) for confirmation and antimicrobial susceptibility testing (AST). The National Antimicrobial Resistance Monitoring System at CDC performed AST on 17 isolates by broth microdilution to determine minimum inhibitory concentrations for 14 antimicrobial agents: amoxicillin/clavulanic acid, ampicillin, azithromycin, ceftiofur, ceftriaxone, cefoxitin, chloramphenicol, ciprofloxacin, gentamicin, nalidixic acid, streptomycin, sulfisoxazole, tetracycline, and trimethoprim/sulfamethoxazole. Results were interpreted using Clinical and Laboratory Standards Institute standards [11] when available.

During the case-control study, we collected 5$10 \mathrm{~mL}$ of blood from each of 20 suspected casepatients, placed the samples into commercial $\mathrm{BD}$ Bactec $^{\text {TM }}$ Aerobic/F media, and transported them to the clinical laboratory at the Makerere College of Health Sciences Department of Medical Microbiology for incubation in the Bactec $9120^{\mathrm{mm}}$ blood culture system. Subcultures onto MacConkey and blood agar were done following instrument signals of growth or at the end of 7 days of incubation. Colonies were identified as $S$. Typhi based on biochemical characteristics including motility, hydrogen sulfide production, fermentation of sugars, urease production, and serological typing characteristics with various specific antisera.

\section{Environmental laboratory investigation}

From 2 to 8 April, juice samples were collected from the Nakasero, Owino, and Shauriyako markets, and $100 \mathrm{~mL}$ water samples were collected from unprotected water sources such as unprotected springs (i.e., underground water sources that do not have barriers protecting them from contamination and run-off) and commercial vendors in Kampala Central Division. We chose these water collection sites because we observed people in the outbreak-affected areas collecting water from these sites. The juice samples were tested because case-persons said they usually consumed these drinks. We collected nine juice samples, including 3 "bushera" (millet and yeast), 2 "munanansi" (pineapple juice with tea leaves), 3 "butunda" (passion fruit), and 1 "bongo" (unpasteurized yogurt drink). We also collected 13 water samples, including 3 "kaveera" (water packaged and sold in a small plastic bag), one unlabeled bottle of water from a street vendor, water from three storage tanks, and water from five unprotected springs. Juice and water samples were tested using a modified version of the United States Environmental Protection Agency's Standard Analytical Protocol for S. Typhi in Drinking Water [12]. Briefly, $125 \mathrm{~mL}$ of specimen was preenriched in $125 \mathrm{~mL}$ of double strength buffered peptone water at $37^{\circ} \mathrm{C}$, followed by parallel enrichment in Selenite Cysteine broth at $37{ }^{\circ} \mathrm{C}$ and RV broth at $42{ }^{\circ} \mathrm{C}$. Cultures from Selenite Cysteine broth were plated onto MacConkey and XLD agars; cultures from RV broth were plated onto XLD agar. All plates were incubated at $37^{\circ} \mathrm{C}$. Plates were inspected at 24 and $48 \mathrm{~h}$ for colony morphology consistent with Enterobacteriacea. Colonies morphologically consistent with $S$. spp. (i.e. lactose negative) were subjected to biochemical testing. Suspect isolates were sent to CDC-Atlanta for biochemical confirmation. For confirmation, suspect cultures were streaked onto Hektoen enteric agar and suspect colonies were subjected to an abbreviated panel of tests, for phenotypic identification of Salmonella or Shigella spp. and biochemical differentiation of $S$. serovars Typhi and Paratyphi A from other Salmonella serovars [10].

\section{Statistical analysis}

Using surveillance data, the attack rates by sex, division, and sex were calculated using population data from the national census [8] and data provided by the Uganda Bureau of Statistics [13]. Using the StatCalc in Epi Info 7 , considering a power of $80 \%$, two sided confidence level of $95 \%$, a case-control ratio of 1:2 with $30 \%$ of cases exposed and $10 \%$ of controls exposed, we would require about 39 cases and 77 controls. To measure the associations between exposure variables and illness status, we used the Mantel-Haenszel method to estimate odds ratios (OR) and their confidence intervals, accounting for frequency-matching of cases and controls. We calculated the proportion of cases and controls who drank 1,2, and 3-4 types of locally made drinks, and used the Chisquare test for linear trend to assess the relationship between the number of types of drinks consumed and odds of illness [14]. 


\section{Results}

\section{Surveillance}

From 17 February to 12 June 2015, we identified 10,230 suspected cases from the six treatment centers established by $\mathrm{MoH}$. Cases were distributed widely throughout Kampala and neighboring areas (Fig. 1).

The epidemic curve of suspected cases suggests that the outbreak started at the beginning of February or perhaps earlier. By the time the outbreak was recognized on 6 February, hundreds of cases had already occurred (Fig. 2). Cases were reported in all five divisions of Kampala: Makindye (32\%, 3234), Rubaga (28\%, 2828), Kawempe $(11 \%, 1144)$, Nakawa $(6.4 \%, 656)$ and Central $(4.2 \%, 428)$; for $19 \%$ (1940) of cases, either no division of residence was identified, or resided outside of Kampala. The attack rate during the outbreak period was highest in Makindye (10/1,000), Rubaga (8.7/1000), and Central
(6.5/1000) Divisions. Males had a higher attack rate than females. The attack rate among people in the 15-59 year age group (12/1000) was 6 times higher than among younger $(2.0 / 1000)$ or older $(2.0 / 1000)$ persons (Table 1$)$.

\section{Case-control study}

In our hypothesis-generating interviews of patients from the area where the outbreak was first identified, consumption of drinks made with water extracted from unprotected sources and packed in unhygienic conditions was often reported.

Of the 33 case-patients we enrolled in the case-control study, $60 \%$ were men; the majority of the case-patients (85\%) were in the 20-39 year and 9.1\% in older age groups. In addition to fever, commonly reported symptoms included abdominal pain $(72.72 \%)$ and headache (69.69\%) (Table 2). We found that $22.58 \%$ (7/31) of case-

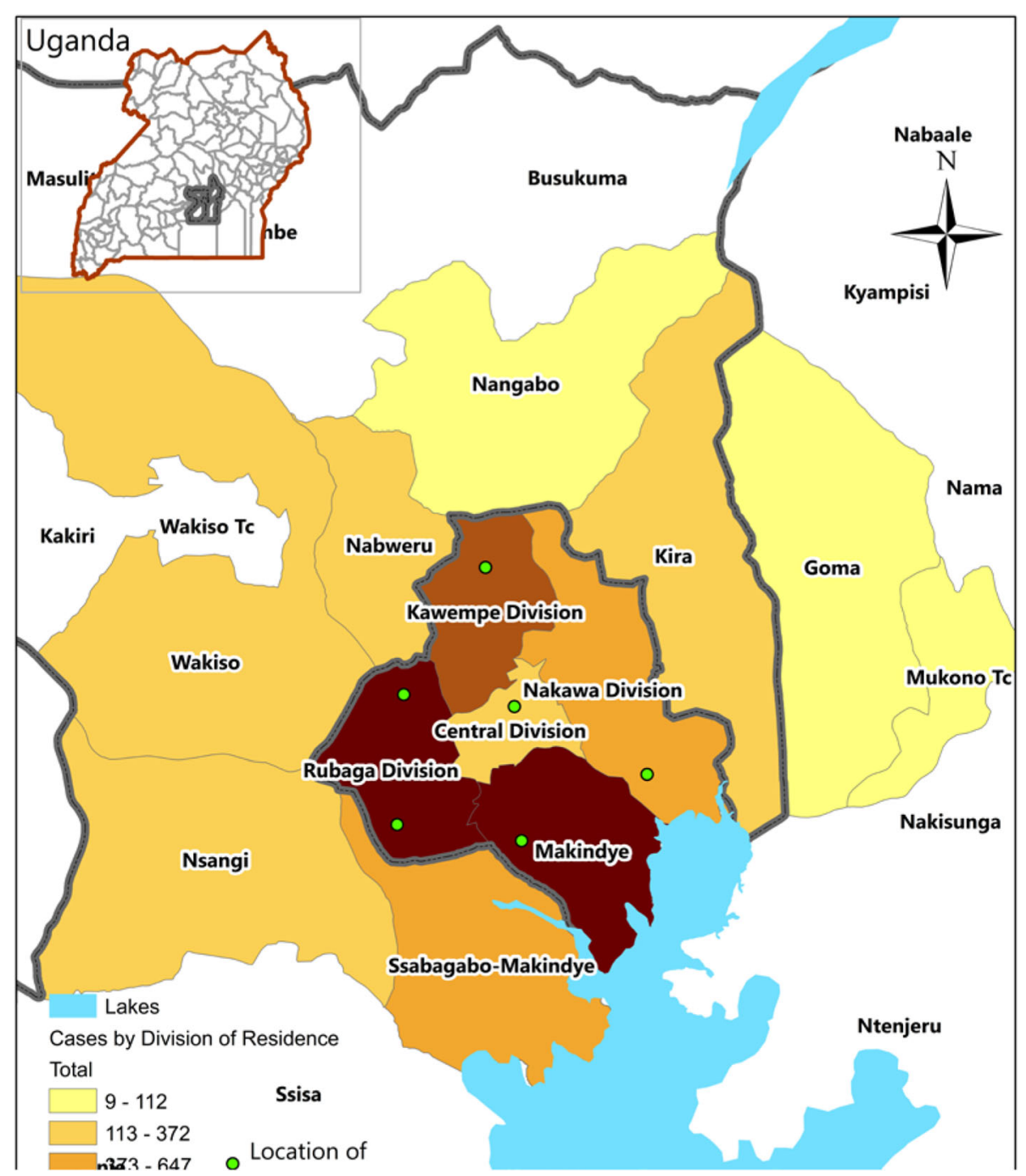

Fig. 1 An intensity map showing treatment centers (green dots) and geographical distribution of suspected case-patients by place of residence during an outbreak of typhoid fever: Kampala, Uganda, $17^{\text {th }}$ February $2015-14^{\text {th }}$ April $2015(n=9325)$ 


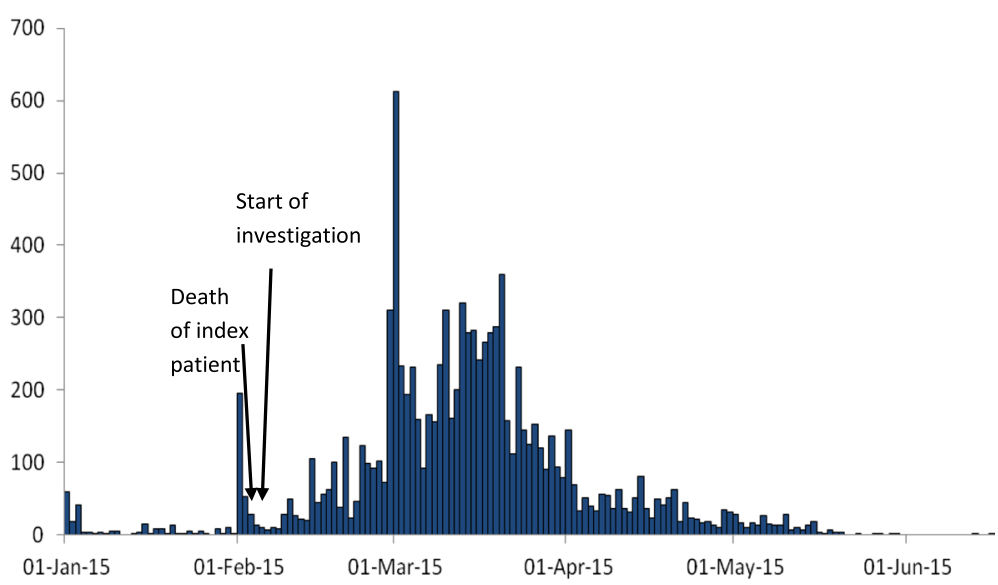

Fig. 2 Distribution of suspected cases of typhoid fever by date of symptom onset during an outbreak of typhoid fever: Kampala, Uganda, 1 January 2015-12 June $2015(n=9515)^{*}$

patients compared with $2.56 \%(2 / 78)$ of controls usually drank locally packaged water in small plastic bags called "kaveera water" $\left(\mathrm{OR}_{\mathrm{M}-\mathrm{H}}=8.90 ; 95 \% \mathrm{CI}=1.60-49.00\right)$; $55 \%(18 / 33)$ of case-patients compared with $19.23 \%$ $(15 / 78)$ of controls drank locally-made passion fruit juice called "butunda" $\left(\mathrm{OR}_{\mathrm{M}-\mathrm{H}}=4.60 ; 95 \% \mathrm{CI}\right.$ : $1.90-$ $11.00) ; 31.25 \%(10 / 32)$ of case-patients compared with $16.67 \%(13 / 78)$ of controls usually drank locally-packed pineapple juice called "munanansi" $\left(\mathrm{OR}_{\mathrm{M}-\mathrm{H}}=2.00 ; 95 \%\right.$ $\mathrm{CI}=0.74-5.20)$; and $15.63 \%$ (5/32) of case-patients compared with $8.97 \%$ (7/78) of controls usually drank cold millet porridge called "bushera" $\left(\mathrm{OR}_{\mathrm{M}-\mathrm{H}}=2.80\right.$; $95 \% \mathrm{CI}=$ 0.76-10.00). Workplace as a source of breakfast $\left(\mathrm{OR}_{\mathrm{M}-\mathrm{H}}\right.$ $=0.25 ; 95 \% \mathrm{CI}=0.07-0.93)$, and workplace as a source of

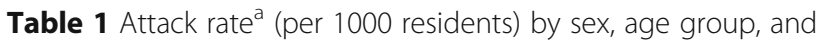
Division of residence during an outbreak of typhoid fever: Kampala, Uganda, 17 February 2015-12 June 2015

\begin{tabular}{ll}
\hline Characteristic & Attack rate (/1000 residents) \\
\hline Sex & 11.00 \\
Male & 8.40 \\
Female & 9.50 \\
Total & \\
Age group (years) & 2.00 \\
$<15$ & 12.00 \\
$15-59$ & 2.00 \\
$\geq 60$ & \\
Division of residence & \\
Central & 6.50 \\
Kawempe & 3.80 \\
Makindye & 10.00 \\
Rubaga & 8.70 \\
Nakawa & 2.30 \\
\hline
\end{tabular}

${ }^{a}$ Based on suspected cases lunch $\left(\mathrm{OR}_{\mathrm{M}-\mathrm{H}}=0.35 ; 95 \% \mathrm{CI}=0.11-1.10\right)$ were not significant risk factors for illness.

When we compared the proportions of case-patients and controls who drank $0,1,2$, or 3-4 types of locallymade drinks, we found that case-patients were more likely to drink multiple types of locally-made drinks than controls (Chi-square for linear trend $=14.65, p<0.001$ ) (Table 3).

\section{Laboratory investigation}

Of 10,230 suspected cases, 3464 (10\%) underwent TUBEX $^{\odot}$ TF testing. Of those, 1038 were positive, representing a positivity rate of $29 \%$. Blood samples from a total of 364 patients (including 20 of 33 case-control study patients) were tested by blood culture and 56 (15\%) (including 5 of the 20 case-control study patients tested) yielded S. enterica ser. Typhi.

Subsequently, 30 of the 56 S. Typhi isolates from blood cultures were confirmed at the U.S. CDC as S. Typhi. CDC determined the minimum inhibitory concentrations for 17 of these isolates, 5 of which were resistant to ampicillin, chloramphenicol, streptomycin, sulfisoxazole,

Table 2 Reported symptoms among case-patients in a casecontrol study during an outbreak of typhoid fever: Kampala, Uganda, 10 February 2015-20 February 2015 ( $n=33)$

\begin{tabular}{lll}
\hline Symptom & Frequency & Percent \\
\hline Fever $\geq 3$ days $^{\mathrm{a}}$ & 33 & 100 \\
Abdominal Pain & 24 & 72.72 \\
Headache & 23 & 69.69 \\
Diarrhea & 10 & 30.30 \\
Nausea or Vomiting & 10 & 30.30 \\
Jaundice & 7 & 21.21 \\
Constipation & 3 & 9.09 \\
\hline a Since 1 January, 2015 & &
\end{tabular}

a Since 1 January, 2015 
Table 3 Dose-response relationship comparing those who consumed 1, 2 and 3-4 implicated drinks to those who did not consume any of the drinks during an outbreak of typhoid fever: Kampala, Uganda, February 2015

\begin{tabular}{llll}
\hline Drinks & \%Cases $(n=33)$ & \%Controls $(n=78)$ & OR \\
\hline 0 & 36 & 64 & 1.0 (Ref) \\
1 & 27 & 26 & $1.90(0.68-5.10)$ \\
2 & 15 & 9.0 & $3.00(0.80-11.00)$ \\
$3-4$ & 21 & 1.0 & $29.00(3.20-260.00)$ \\
\hline
\end{tabular}

nalidixic acid, trimethoprim/sulfamethoxazole and had intermediate interpretation to ciprofloxacin. The remaining 12 were resistant to nalidixic acid and had intermediate interpretation to ciprofloxacin.

\section{Environmental investigation}

One of 3 "kaveera water" samples and the unlabeled bottle of water sold by street vendors contained lactose fermenting bacteria, which are commonly Enterobacteriaceae and associated with fecal contamination. The 2 other "kaveera water" samples contained non-lactose fermenting bacteria, also consistent with fecal contamination, and one isolate was further identified as non-typhoidal Salmonella. The 5 water samples from unprotected springs showed evidence of robust contamination with lactose fermenting bacteria. Lactose non-fermenting colonies from 2 spring water samples were identified as non-typhoidal Salmonella spp. Lactose fermenting bacteria were also detected in 2 of 3 "bushera" samples, 1 of 2 "munanasi" samples, all 3 "munanansi" juice samples, and the "bongo" sample. Additionally, non-typhoidal Salmonella was cultured from 1 "bushera", 1 "munanansi", and 1 passion fruit juice sample.

\section{Discussion}

Our investigation revealed a prolonged and widespread outbreak of typhoid fever that affected thousands of people in all five divisions of Kampala City over several months. Contaminated water from unprotected sources and drinks made with it were the likely vehicles of infection early in the outbreak. Juice and water samples obtained from street vendors and water samples collected from unprotected spring water sources showed evidence of fecal contamination. Although $S$. Typhi was not recovered from environmental testing, non-Typhi Salmonella were isolated from five street-vended beverage samples from the implicated markets.

All 17 isolates of $S$. Typhi from blood tested at CDC were resistant to nalidixic acid and had intermediate interpretation to ciprofloxacin. It is possible that persons affected by the antibiotic-resistant strains during this outbreak had experienced complications of typhoid fever considering that antibiotic-resistant strains of $S$. Typhi are associated with more severe form of the illness, complications and death [15].

This outbreak may have started in January 2015 or even earlier; however, it was not recognized until early February because routine clinical and laboratory surveillance systems for typhoid fever were not in place before the investigation. The widespread nature of the outbreak is compatible with a waterborne source. The sudden increase in cases after the start of the investigation was likely due to active community outreach and education about the symptoms of typhoid fever and the availability of prompt, free diagnostic testing and treatment through newly established treatment centers. The gradual decline in cases from mid-March onwards was likely the result of patient treatment and public health interventions including provision of free water chlorination products, sensitization of residents on water treatment, and the establishment of free alternative safe water sources in the most affected communities. Based on the evidence we presented, the Kampala Capital City Authority sealed off all underground water sources and worked with the National Water and Sewerage Corporation to ensure the provision of accessible alternative sources of water to the affected communities.

In Uganda, as in many low and middle income countries, definitive diagnostic tests for typhoid fever such as blood culture are usually unavailable, unaffordable, or inconsistently applied [16]. Instead, typhoid fever diagnosis and surveillance often rely on clinical judgment or on the Widal test, which has poor sensitivity and specificity [17]. Moreover, physicians often give presumptive antibiotic and/or antimalarial treatment for febrile illnesses $[18,19]$ without attempting to determine the etiology. Previous studies have indicated that a significant proportion of febrile illness in Uganda is caused by bacteremia, including invasive non-Typhi salmonellosis and typhoid fever [20]. A more robust approach in these settings could entail periodically identifying persons with febrile illness in the communities and taking blood culture for confirmation [21]. The blood samples could be collected and sent using a specialized transport network to regional laboratory centers around the country where confirmative tests can be performed. This system has been successfully used to improve diagnostic services in early infant HIV/AIDS diagnosis [22]. Sentinel surveillance for febrile illnesses based on blood cultures would accelerate the early identification of outbreaks and implementation of control measures.

Waterborne typhoid and paratyphoid fever affect an estimated 27 million people worldwide each year [3]. In developing countries, where safe water and sanitation systems have not been well-established, large-scale typhoid and paratyphoid outbreaks sometimes occur [7, 23-26]. During a previous typhoid outbreak in Kasese and Bundibugyo 
districts, Uganda, in 2009-2011, which affected 8092 persons, the vehicle of transmission was also found to be unclean water [7]. The current outbreak was likely caused by consuming contaminated water from unprotected ground water sources. Kampala city has more than 200 unprotected ground water sources, most of which serve as unprotected sources of water for economically disadvantaged people in the city such as those in our investigation [27]. Unsafe disposal of excreta and solid waste are significant factors that contribute to contamination of ground water in Kampala [28]. This outbreak investigation highlights the importance of ensuring access to affordable, safe, treated drinking water and improved sanitation and waste management systems for resource-constrained urban populations.

Risk factors for typhoid transmission were not assessed later during this outbreak, when foodborne transmission might have become more common. Recurrent contamination of unprotected water sources with $S$. Typhi likely continued to sustain the outbreak propagation over the course of several months.

According to the Uganda Demographic Health Survey 2011 [29], almost 30\% of people living in urban areas and more than $60 \%$ of those living in rural areas do not treat their water before drinking it. Barriers to safer drinking water include the cost associated with establishing a piped treated water system or purchasing water treatment products for household use and the false perception that naturally occurring water sources could be safe [30].

In the aftermath of outbreaks like this one, public health authorities face 3 possible options: The first option is to do nothing but respond to outbreaks as they occur. Governments in resource-constrained settings often choose this option, leaving the population vulnerable to outbreaks of waterborne diseases including cholera, hepatitis A and E, cryptosporidiosis, shigellosis, and many others in addition to typhoid and paratyphoid fever. The second option is mass vaccination against typhoid fever. A cost-effectiveness evaluation of a hypothetical typhoid vaccination campaign was carried out after the multi-year outbreak of typhoid fever in Kasese District, Uganda, and it was estimated to be highly costeffective [31]. However, vaccination against the many different pathogens that cause waterborne diseases is not possible because vaccines are not yet available for many of them (e.g. cryptosporidiosis, shigellosis, paratyphoid fever, etc.). In addition, typhoid fever vaccines have been shown to have varied levels of effectiveness (from 50 to 95\%) and to last for varied lengths of time (from 3 to 10 years) [15].

The third and final option is to improve the water and sanitation systems. Improvement of sanitation, hygiene and clean water supply around the world could avert $\geq 90 \%$ of diarrheal disease episodes annually [32]. In
North America and Europe, typhoid fever caused largescale outbreaks from the late $19^{\text {th }}$ through the early $20^{\text {th }}$ century [33, 34]. After improvement of municipal water and sanitation systems in the early $20^{\text {th }}$ century, waterborne outbreaks of communicable diseases including typhoid fever drastically decreased [35-37]. Improved sanitation measures such as having a basic pit latrine or a toilet connected to a septic tank curtail the direct contact between human waste and water or the environment. Yet in 2012, only 33\% of the urban population in Uganda had access to adequate sanitation, an increase of only $1 \%$ since 1990 , and $2 \%$ still practiced open defecation [38]. Although improving water and sanitation systems requires a substantial investment by the government, ultimately it is highly cost-effective in the reduction of many waterborne diseases [39].

\section{Strengths and limitations}

A major limitation of our investigation was that, due to inadequate laboratory capacity to confirm a large number of cases early in the outbreak, and the need to rapidly identify the mode of transmission to inform effective interventions, we included non-laboratory confirmed cases in our case-control study. While a clinical case definition for typhoid fever cases can lead to misclassification, and is not recommended during nonoutbreak situations, during an outbreak such a case definition will often perform well, as measured by good positive and negative predictive values [40]. Also, the information on usual sources of water and food was based on self-reports, which could represent a source ofinformation bias. Another limitation is that data on mortality or on complications such as intestinal perforations were not collected. With over 10,000 cases it is likely that there were intestinal perforations and deaths but no surveillance for those outcomes was done. A study is currently being conducted to assess these severe impacts of this outbreak. In addition, only a few water and juice samples were tested, which could explain why S. Typhi was not isolated during the environmental investigation. Also, water and juice samples were tested using the reagents and procedures that were available in the laboratory for testing clinical specimens for Salmonella, and not more conventional methods for evaluating the potential presence of fecal contamination in these types of samples.

\section{Conclusion}

In conclusion, this investigation revealed a large outbreak of typhoid fever that affected thousands of people in Kampala, Uganda, which appeared to have been caused by consuming contaminated water and local drinks made from it. To prevent future waterborne outbreaks, we recommended that the Kampala Capital City Authority, the 
$\mathrm{MoH}$, the National Water and Sewerage Corporation, and partners invest in improving access to potable water, and safe sanitation and hygiene facilities [41].

\section{Additional file}

Additional file 1: The questionnaire that was used in the collection of information from cases and controls is available as a supplementary file called Typhoid questionnaire. It provides details on which questions were asked about food and water exposures. (DOC 87 kb)

\section{Abbreviations}

CDC: United States Centers for Disease Control and Prevention;

MoH: Ministry of Health; WHO: World Health Organization

\section{Acknowledgements}

The authors would like to acknowledge Mr. James Oweka and Tobias Vudriko of Medical Research Council Uganda, Dr Robert Downing of Uganda Virus Research Institute, Dr. Alex Ario Riolexus of the Uganda Public Health Fellowship Program and health inspectors of Kampala Capital City Authority for their valuable contributions to this work. We also acknowledge the numerous personnel from the Central Public Health Laboratories, and members of the Uganda People's Defense Forces for their assistance in the investigation.

\section{Funding}

Authors were funded by their respective organizations. This work was also funded by the Cooperative Agreement - Provision of Comprehensive HIV/ AIDS services and Developing National Capacity to manage HIV/AIDS Programs in the Republic of Uganda under the President's Emergency Plan for AIDS Relief (grant number 5U2GGH000817-03) with the Centers for Disease Control and Prevention. The funders had no role in study design, data collection and analysis, decision to publish, or preparation of the manuscript.

\section{Availability of data and materials}

Data will not be shared. On receipt of the data, the authors agreed not to reproduce or distribute them. As recipients, the authors were permitted to publish any analyses of the data, but not the data itself subject to confidentiality conditions.

\section{Author contributions}

SNK, LB, FN, GP, DWO, RM, CK, BK, BM and AEO were involved in the design and implementation of the investigation. $\mathrm{HK}, \mathrm{PH}$, and JNS were involved in the implementation of the clinical laboratory component of the investigation. IM, SK, SK, MD were involved in the implementation and data collection and collation. $\mathrm{VRH}$ and $\mathrm{AB}$ were involved in the environmental laboratory implementation of the investigation. EM, JR and B-PZ were involved in the data analysis. SNK, EM and B-PZ had primary responsibility for final content. All authors participated in the writing, read and approved the final manuscript.

\section{Competing interests}

The authors declare that they have no competing interests.

\section{Consent for publication}

Not applicable.

\section{Ethics approval and consent to participate}

The Ministry of Health of Uganda gave the directive and approval to investigate this outbreak. In agreement with the International Guidelines for Ethical Review of Epidemiological Studies by the Council for International Organizations of Medical Sciences (1991), the Office of the Associate Director for Science, CDC/Uganda, determined that this activity was not human subjects research and its primary intent was public health practice or a disease control activity (specifically, epidemic or endemic disease control activity). Verbal informed consent was obtained from the participants before the start of each interview. Written consent could not be obtained because it was impractical as most of the participants were illiterate. However, the purpose and nature of the investigation were explained to all participants. Participants were also informed that their involvement was entirely voluntary and their refusal to answer any or all of the questions would not result in any negative consequences. Participants identified as patients were referred for free treatment at the designated treatment centres. To protect participants' confidentiality, personal information were de-identified during data analysis, and the interview forms were locked up.

\section{Disclaimer}

The views expressed in this article are those of the authors and do not necessarily represent the official position of the Centers for Disease Control and Prevention.

\section{Author details}

${ }^{1}$ Uganda Public Health Fellowship Program, Field Epidemiology Track, Ministry of Health, Kampala, Uganda. ${ }^{2}$ Makerere University College of Health Science Microbiology Laboratory, Kampala, Uganda. ${ }^{3}$ Makerere University School of Public Health, Kampala, Uganda. ${ }^{4}$ Public Health Emergency Operations Center, Ministry of Health, Kampala, Uganda. ${ }^{5}$ Medical Research Council, Kampala, Uganda. ${ }^{6}$ Epidemiology and Surveillance Division, Ministry of Health, Kampala, Uganda. ${ }^{7}$ US Centers for Disease Control and Prevention, Atlanta, Georgia, USA. ${ }^{8}$ US Centers for Disease Control and Prevention, Kampala, Uganda.

Received: 13 May 2016 Accepted: 23 December 2016 Published online: 05 January 2017

\section{References}

1. Heymann DL. In: Heymann DL, editor. Control of Communicable Diseases. An Official Report of the American Public Health Association. 20th ed. United States of America: Alpha Press; 2014.

2. World Health Organization. The diagnosis, treatment and Prevention of Typhoid Fever. World Health Organisation, Department of Vaccines and Biologicals: CH-1211 Geneva 27; 2003. WHON\&B/03.07

3. Crump JA, Luby SP, Mintz ED. The global burden of typhoid fever. Bull World Health Organ. 2004;82:346-53.

4. Centers for Disease Control. Typhoid Fever 2013. Available from: https:// www.cdc.gov/typhoid-fever/index.html. Accessed 27 July 2015.

5. Mogasale V, Maskery B, Ochiai RL, Lee JS, Mogasale W, et al. Burden of typhoid fever in low-income and middle-income countries: a systematic literature-based update with risk-factor adjustment. Lancet Glob Health. 2014:2:e570-80

6. Neil KP, Sodha SV, Lukwago L, O-Tipo S, Mikoleit M, Simington SD, et al. A Large Outbreak of Typhoid Fever Associated With a High Rate of Intestinal Perforation in Kasese District, Uganda, 2008-2009. Clin Infect Dis. 2012;54: 1091-9.

7. Walters MS, Routh J, Mikoleit M, Kadivane S, Ouma C, et al. Shifts in Geographic Distribution and Antimicrobial Resistance during a Prolonged Typhoid Fever Outbreak — Bundibugyo and Kasese Districts, Uganda, 2009-2011. PLoS Negl Trop Dis. 2015;8(3):e2776-e26.

8. Uganda Bureau of Statistics. National Population and Housing Census 2014 Revised Edition. Kampala: 2014. Available from http://www.ubos.org/ onlinefiles/uploads/ubos/NPHC/ 2014\%20National\%20Census\%20Main\%20Report.pdf. Accessed 12 Jan 2016.

9. Tam FCH, Ling TKW, Wong KT, Leung DTM, Chan RCY, Lim PL. The TUBEX test detects not only typhoid-specific antibodies but also soluble antigens and whole bacteria. J Med Microbiol. 2008:57:316-32.

10. Mikoleit ML. World Health Organization Foodborne Infections Network Laboratory Protocol: "Biochemical Identification of Salmonella and Shigella Using and Abbreviated Panel of Tests.". 2010.

11. Clinical and Laboratory Standards Institute. Performance Standards for antimicrobial susceptibility testing; twenty second informational supplement M-100-S-25. Clinical and Laboratory Standards Institute. Wayne; 2015. Available from http://shop.clsi.org/site/Sample_pdf/M100S25_sample. pdf. Accessed 8 Feb 2016.

12. Sanjiv RS. Standard Analytical Protocol for Salmonella Typhi in Drinking Water. 600/R - 10/133. Washington, DC: United States Environmental Protection Agency, Office of Research and Development.

13. Uganda Bureau of Statistics. 2014 Statistical Abstract. 2014. Available from http://www.ubos.org/onlinefiles/uploads/ubos/statistical_abstracts/ Statistical_Abstract_2014.pdf. Accessed 14 Sept 2015. 
14. BMJ Publishing Group Ltd. About The BMJ: Copyright @ 2015 BMJ Publishing Group Ltd; 2015. [cited 2015 12th August 2015]. 8. The Chi Squared Tests]. Available from: http://www.bmj.com/about-bmj/resourcesreaders/publications/statistics-square-one/8-chi-squared-tests.

15. World Health Organization, Weekly Epidemiological Record, in Typhoid Vaccines: WHO Position Paper. Geneva: World Health Organization; 2008. p. 49-60.

16. Archibald LK, Reller LB. Clinical microbiology in developing countries. Emerg Infect Dis. 2001;7:302-5.

17. Keddy KH, Sooka A, Letsoalo ME, Hoyland G, Chaignat CL, Morrissey AB, et al. Sensitivity and specificity of typhoid fever rapid antibody tests for laboratory diagnosis at two sub-Saharan African sites. Bull World Health Organ. 2011;89(9):640-7.

18. Mukonzo JK, Namuwenge PM, Okure G, Mwesige B, Namusisi OK, Mukanga D. Over-the-counter suboptimal dispensing of antibiotics in Uganda. J Multidiscip Healthc. 2013;6:303.

19. Batwala V, Magnussen P, Nuwaha F. Antibiotic use among patients with febrile illness in a low malaria endemicity setting in Uganda. Malar J. 2011; 10:377.

20. Kibuuka A, Byakika-Kibwika P, Achan J, Yeka A, Nalyazi JN, Mpimbaza A, et al. Bacteremia Among Febrile Ugandan Children Treated with Antimalarials Despite a Negative Malaria Test. Am J Trop Med Hyg. 2015;93(2):276-80.

21. Crump JA, Mintz E. Global trends in typhoid and paratyphoid fever. Clin Infect Dis. 2010;50(2):241-6.

22. Kiyaga C, Sendagire H, Eleanor J, McConnell I, Grosz J, Narayan V, et al Uganda's new national laboratory transport system: A successful model for improving access to diagnostic services for early infant HIV diagnosis and other programs. PLoS One. 2013;8(11):e78609.

23. Polonsky JA, Martinez-Pino I, Nackers F, Chonzi P, Manangazira P, Herp MV, et al. Descriptive Epidemiology of Typhoid Fever during an Epidemic in Harare, Zimbabwe, 2012. PLoS One. 2014;9(12):e114702.

24. Muyembe-Tamfum JJ, Veyi J, Kaswa M, Lunguya O, Verhaegen J, Boelaert M An outbreak of peritonitis caused by multidrug-resistant Salmonella Typhi in Kinshasa, Democratic Republic of Congo. Travel Med Infect Dis. 2009;1:40-3.

25. Mermin JH, Villar R, Carpenter J, Roberts L, Samaridden A, Gasanova L, et al. Massive Epidemic of Multidrug-Resistant Typhoid Fever in Tajikistan Associated with Consumption of Municipal Water. J Infect Dis. 1999;179(6): $1416-22$.

26. Lewis MD, Serichantalergs O, Pitarangsi C, Chuanak N, Mason CJ, Regmi RL, et al. Typhoid Fever: A Massive, Single-Point Source, Multidrug-Resistant Outbreak in Nepal. Clin Infect Dis. 2005;40(4):554-61.

27. Howard G, Pedley S, Barrett M, Nalubega M, Johal K. Risk factors contributing to microbiological contamination of shallow groundwater in Kampala, Uganda. Water Res. 2003;37(14):3421-9.

28. Matagi S. Some Issues of Environmental Concern in Kampala, the Capital City of Uganda. Environ Monit Assess. 2002;77(2):121-38.

29. Uganda Bureau of Statistics (UBOS), ICF International Inc. Uganda Demographic Health Survey 2011. Kampala Uganda: UBOS and Calverton, Maryland: ICF International Inc, 2012

30. Merkel L, Bicking C, Sekhar D. J Community Health. 2012;37(1):195-201.

31. Carias C, Walters MS, Wefula E, Date KA, Swerdlow DL, Vijayaraghavan M, et al. Economic evaluation of typhoid vaccination in a prolonged typhoid outbreak setting: the case of Kasese district in Uganda. Vaccine. 2015;33(17): 2079-85.

32. Evans B. Securing Sanitation - The Compelling case to address the crisis. Stockholm International Water Institute; 2005. Available at http://eprints. whiterose.ac.uk/id/eprint/42856. Accessed 17 June 2015.

33. Wolman A, Gorman AE. The significance of waterborne typhoid fever outbreaks 1920-1930. J Am Water Works Assoc. 1939:23(2):160-201.

34. Hunter P. Waterborne Disease: Epidemiology and Ecology. Chinchester: Wiley; 1997.

35. Cutler D, Miller $\mathrm{G}$. The role of public health improvements in health advances: the twentieth-century United States. Demography. 2005;4(2):1-22.

36. Japanese Ministry of Health and Welfare. A 50-year history of the Ministry of Health and Welfare. Editorial Board of the 50 year history of the ministry of Health and welfare. Japan: 1988.

37. Centers for Disease Control. Achievements in Public Health, 1900-1999: Control of Infectious Diseases. MMWR Morb Mortal Wkly Rep. 1999;48(29):621-9.

38. World Health Organization (WHO), United Nations Childrens Fund (UNICEF). Progress on Drinking water and sanitation 2014 Update. 2014.
39. Clasen T, Cairncross S, Haller L, Bartram J, Walker D. Cost-effectiveness of water quality interventions for preventing diarrhoeal disease in developing countries. J Water Health. 2007;5(4):599-608.

40. Sharma SK. Importance of Case Definition in Epidemiological studies. Neuroepidemiology. 2011;37:141-2.

41. Clasen T, Schmidt WP, Rabie T, Roberts I, Cairncross S. Interventions to improve water quality for preventing diarrhea: systematic review and meta analysis. BMJ. 2007;334(7597):782

\section{Submit your next manuscript to BioMed Central and we will help you at every step:}

- We accept pre-submission inquiries

- Our selector tool helps you to find the most relevant journal

- We provide round the clock customer support

- Convenient online submission

- Thorough peer review

- Inclusion in PubMed and all major indexing services

- Maximum visibility for your research

Submit your manuscript at www.biomedcentral.com/submit

) Biomed Central 